\title{
Magnetic diffusion: Scalability, reliability, and QoS of data dissemination mechanisms for wireless sensor networks
}

\author{
Hsing-Jung Huang, Ting-Hao Chang, Shu-Yu Hu, Polly Huang * \\ Electrical Engineering Department, National Taiwan University, No. 1, Sec. 4, Roosevelt Road, Taipei, Taiwan
}

Available online 3 March 2006

\begin{abstract}
Envisioning a new generation of sensor network applications in healthcare and workplace safety, we seek mechanisms that provide timely and reliable transmissions of mission-critical data. Inspired by the physics in magnetism, we propose a simple diffusion-based data dissemination mechanism, referred to as the magnetic diffusion (MD). In that, the data sink, functioning like the magnet, propagates the magnetic charge to set up the magnetic field. Under the influence of the magnetic field, the sensor data, functioning like the metallic nails, are attracted towards the sink. We compare MD to the state-of-the-art mechanisms and find that MD: (1) performs the best in timely delivery of data, (2) achieves high data reliability in the presence of network dynamics, and yet (3) works as energy efficiently as the state of the art. These suggest that MD is an effective data dissemination solution to the mission-critical applications.
\end{abstract}

(C) 2006 Elsevier B.V. All rights reserved.

Keywords: Sensor network; Data dissemination

\section{Introduction}

The technological advances have enlightened a future of intelligent and pervasive computing and communication. In that, miniature and robust sensor nodes would be able to generate, pre-process, and communicate metrics about the environment. For instance, auto-sensing of the room temperature allows tuning of the air conditioning to the level just as necessary. Auto-detection of pulse anomalies allows prevention of irreversible damages caused by diseases that are preceded by arrhythmia.

The emergence of the sensors and sensor networks can impact a wide variety of applications from national security to consumer electronics. Envisioning a new generation of sensor network applications in healthcare and workplace safety, we seek mechanisms that provide reliable and timely transmissions of mission-critical data. Even though wall power will be more accessible in consumer electronics applications, the sensor nodes are likely to run

\footnotetext{
${ }^{*}$ Corresponding author.

E-mail addresses: r93921046@ntu.edu.tw, r93921093@ntu.edu.tw, b89117@csie.ntu.edu.tw, phuang@cc.ee.ntu.edu (P. Huang).
}

on limited battery power for the ease of deployment. Thus, energy efficiency remains an important design challenge.

Much of the related work in sensor network data dissemination either emphasizes the energy-efficient design or stresses the reliable data transfer. We have not yet been able to identify any mechanism comparable to the proposed mechanism, magnetic diffusion (MD), that aims at achieving timely delivery, reliability, and energy efficiency.

MD is a simple data dissemination mechanism that promises all the above properties. The inspiration comes from the magnetic interactions in the nature. Consider the data sink as a magnet and the data as nails. The data will be attracted towards the sink according to the magnetic field just as the metallic nails being attracted towards the magnet. The magnetic field is established by setting up the proper magnetic charges on the sensor nodes within the range of data sink. The strength of the charge is determined by the hop distance to the sink and the level of resource available at the sink. The data will be propagated based on the magnetic field from low to high magnetically charged nodes. This way of disseminating data results in optimal delay multi-path forwarding. 
We are able to observe from the simulation results that MD does (1) perform the best in timely delivery of data, (2) achieve high data reliability in the presence of network dynamics, and yet (3) work as energy efficiently as the state-of-the-art mechanisms. We thus conclude that $\mathrm{MD}$ is a promising data dissemination solution to the mission-critical applications.

\section{Related work}

There is a significant volume of related work in sensor network data dissemination. MD is particularly related to research in energy-efficient routing and reliable data delivery. To put our work in context, we compare and contrast the existing work to our mechanism. In general, we are not able to identify any mechanisms comparable to MD that aims at achieving timely delivery, reliability, and energy efficiency. This is primarily due to the limited attention in sensor network design for mission-critical applications.

We choose to compare MD to the mechanisms that we are able to find actual implementations for the frequently used Motes/TinyOS platform [28,29]. Directed diffusion [1,2] and flooding are the mechanisms considered in our evaluation. To facilitate discussions in the later sections, we give an overview of the directed diffusion mechanism, following the summary of existing work.

\subsection{Energy-efficient data dissemination}

There are three major classes of energy-efficient data dissemination mechanisms, the cluster-based, randomwalk, and location-aware mechanisms. The cluster-based approaches [4-6] evenly distribute energy load among the sensors in the network. The idea is to form clusters for localized data dissemination and send only the aggregated data across the clusters to conserve energy. Random-walk approaches $[7,8]$ randomly select the next hop targets to disseminate data. These mechanisms are energy efficient in that they avoid the sending of control messages and the maintenance of routing states. Location-aware schemes $[9,10]$ exploit the nodes' geographical location information to minimize the cost of disseminating data. The location information might not be easy to obtain for certain sensor network applications.

More closely related to our work are the mechanisms dealing with efficient data dissemination to mobile sinks $[11,12]$. MD is similar in that our mechanism also aims at energy-efficient data dissemination for dynamic networks. $\mathrm{MD}$ is yet different for that the mechanism design takes into account mobile non-sink nodes and considers the on-off type of network dynamics.

\subsection{Reliable data dissemination}

There are two major approaches towards better data delivery reliability. The passive approach retransmits data when losses are detected, whereas the active approach aims, instead, at avoiding losses.

More specifically in the passive approach category, some proposed mechanisms $[13,14]$ recover data end-to-end, and the others $[15,16]$ retransmit hop-by-hop. In the end-to-end mechanisms, the sink nodes track the status of data delivery and send request messages to other nodes for recovery. The hop-by-hop mechanisms implement the data recovery between two neighboring nodes. When a data packet is lost, the intermediate sending node retransmits.

In the active approach category, several schemes [17-19] attempt to improve reliability by avoiding congestions. By avoiding congestions, these schemes indirectly lower the loss rate and improve the overall reliability. In addition, some schemes [20-22] avoid losses by selecting the data paths of lower loss rates.

Our work is closely related to [23], a multi-path mechanism. This work has suggested that disseminating data over multiple paths improves the reliability. The number of paths selected in the mechanism depends on the priority of the data and the network situation. Our MD is similar to this mechanism in that we also disseminate data over multiple paths for reliability. The primary difference is in our choices of the multiple paths. Opting for energy efficiency and timeliness of data delivery, we scope our choice to all the available shortest paths.

\subsection{Directed diffusion}

Directed diffusion $[1,2]$ is a data-centric data dissemination protocol for wireless sensor networks. The mechanism achieves energy efficiency by means of selecting empirically good paths and in-network aggregation. There are two dissemination modes in directed diffusion: one-phase pull and two-phase pull.

\subsubsection{One-phase pull}

In one-phase pull (OPP), the sink periodically broadcasts an interest message to each of its neighbors. The interest message specifies the data the sink is interested. The neighboring nodes continue to broadcast the interest message to their own neighbors if the interest message is not a duplicate. This action will be repeated until all nodes have received the interest message. Then, the source with the matching data selects the path of shortest latency according to the interest arrival time, and disseminates data along this path to the sink. The data path is periodically refreshed by interest messages.

\subsubsection{Two-phase pull}

Two-phase pull (TPP) operates in a similar way with a slight complication. The sink also periodically broadcasts an interest message to its neighbors, and the neighbors operate similarly to those in OPP. The main difference is at the broadcasting of exploratory data from the data source to all nodes in the sensor network. After the sink receives this exploratory data packet, it reinforces a good 
path back upstream by sending a reinforcement message. This reinforced path is determined according to metrics such as delay, loss rate, and available bandwidth. The node that receives the reinforcement message acts in the same way and forwards the reinforcement message back upstream until the source receives this reinforcement message. After that, the source sends data along the reinforced path.

To adapt to network dynamics, the reinforced path is periodically refreshed by periodic exploratory data. To conserve energy due to the flooding of data packets, the interval of periodic exploratory data is set to be significantly longer than that of the periodic interest messages.

Please note following important differences between OPP and TPP that will be referred later while comparing the performance of OPP, TPP, and MD:

- To avoid collisions, TPP implements its own random wait mechanism to prevent synchronized sending of exploratory data. Each node selects a random waiting time when receiving an exploratory data. There is only one data transmission path in OPP. It does not need to schedule a random wait before propagating data.

- To adapt to failures, OPP refreshes the data path by periodic interest message, whereas TPP refreshes the reinforced path by periodic exploratory data.

- The frequencies of sending exploratory data and interest messages are different. The frequency of periodic exploratory data is twice as low as that of periodic interest messages.

\section{Magnetic diffusion}

Magnetic diffusion is simple and yet powerful. Consider the data sink as a magnet and the data as metallic nails. The data will be attracted towards the sink according to the magnetic field just as the nails are attracted towards the magnet. The magnetic field is established by setting up the proper magnetic charges on the sensor nodes within the magnetic influence of the data sink. The strength of the charge is determined by both the hop distance to the sink and the level of resource available on the sink. The data will be propagated based on the magnetic field from lowcharge to high-charge nodes (Fig. 1). This way of disseminating data promises the following properties.

Multiple paths. By the simple principle of data traveling towards the center of attraction, the sensor nodes forward data coming strictly from the nodes with lower charges (Fig. 2). Forwarding data this way, the paths selected by MD are optimal. Furthermore, there can be multiple next hops to forward the data. Thus, MD sends data in an optimal multi-path fashion.

Load balancing. When there are multiple magnets in the environment, the multiple magnetic fields will find their balance. The nails will thus be attracted by the magnets nearby. The same principle applies to MD. When there exist multiple sinks, their magnetic influences, decrement-

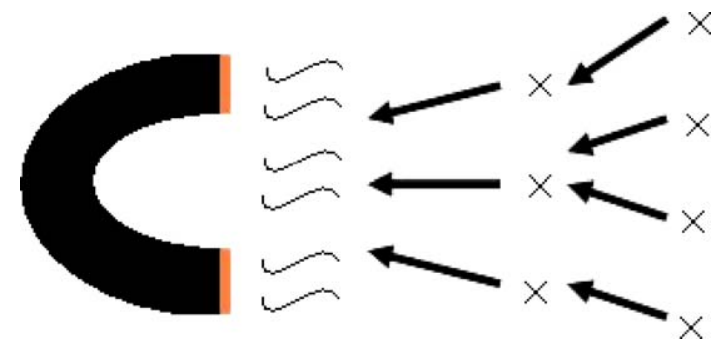

Fig. 1. Data moving towards the magnet (i.e., the sink).

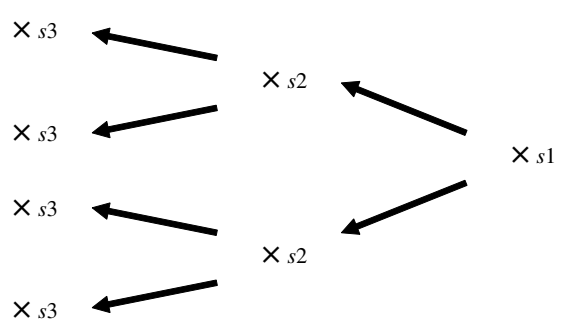

Fig. 2. Multiple paths: multiple nodes with equal charge, $s 1<s 2<s 3$.

ing MD charges from the sinks, will strike a balance (Fig. 3) and attract the data nearby. One advantage of such property is that whenever a new sink joins or an old sink leaves this system, a new balance will be re-established automatically.

Resource awareness. In the real world, some data sinks might be more resource-limited than the others. For example, there could be sink nodes supplied by unlimited wall power, while others run on batteries. By assigning sink charges based on the amount of resource at hand, we can achieve the goal of balancing the use of heterogeneous resource and potentially prolonging the network lifetime without human intervention.

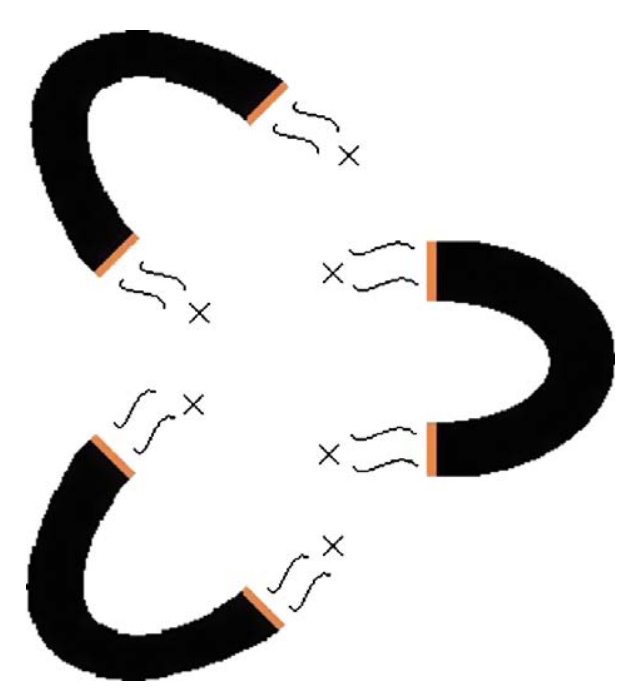

Fig. 3. Load balancing: multiple magnets attracting nearby nails. 
In a healthcare example, a patient with chronic diseases may wear biometric sensors for long-term monitoring of the patient's body temperature, blood pressure, and heart rate. These sensor data need to be collected to a remote patient record server for further analysis. When the patient goes outdoors where there are no infrastructural sinks, the sensor data will need to be transmitted to a resource-limited personal device such as the patient's PDA. Through the GPRS interface, the sensor data can be transmitted to the patient record server. When the patient comes back home, or wherever there are more resourceful sinks such as a fullfledged PC with low-cost ADSL connection, one can assign a stronger magnetic charge to the PC. Then the data, aware of the stronger attraction, will go through the PC rather than the PDA (Fig. 4). Such resource-aware feature brings not only convenience but also optimized utilization of the resources in the environment.

MD operates in two phases: the interest broadcast and data propagation. The magnetic field is established in the interest broadcast phase such that the data can be disseminated towards the sink in the data propagation phase. In the subsections below, we describe the details of the MD operation and the implementation options.

\subsection{Interest broadcast}

We first introduce two data entities involved in the interest broadcast phase: the interest and the entry. The interest is a message used to establish the magnetic field to facilitate the proper flow of data toward the sink. An interest message consists of two elements, the data type and magnetic charge. The former records what data type the sink wants to collect. The latter is an integer value recording the strength of the magnetic influence from the sink. The entry is where a node records the data type and the magnetic charge in each node.

When a sink wants to collect data, it sends an interest message to its neighbors. When a node receives an interest message for the first time, it creates an entry for this interest. Then, the node decrements the magnetic charge in the interest by one. The node records the data type and the magnetic charge into its entry and then forwards the interest message to its neighbors. The decrementing magnetic charges from the sink to source will guide the flow of data in the reverse direction, mimicking the traverse of metallic nails from the low-charge to high-charge points in the magnetic field.

If a node receives an interest message and the corresponding entry exists, it will compare the decremented magnetic charge of the interest with the one in the entry. If the magnetic charge of the interest is greater than the one in the entry, the node will update the value in the entry to that of the interest and forward the interests to its neighbors. Otherwise, the node will discard the interest knowing the interest is not from a node closer to the current sink.

The sink node will broadcast interest messages periodically to re-establish proper magnetic charges in case of network dynamics. It provides a robust environment especially for the dynamic network.

In Fig. 5(a), the sink node broadcasts the interest to its neighbor nodes. In Fig. 5(b), both nodes, A and B, receive the interest, create corresponding entries, set the data type and proper magnetic charges in the entries, and then propagate the interests to their neighbors. In Fig. 5(c), node C, $\mathrm{D}$, and $\mathrm{E}$ receive the interests from node $\mathrm{A}$ and $\mathrm{B}$ and repeat the actions taken by A and B in Fig. 5(b). Each node may receive and discard duplicate interests or interests of weaker magnetic charges.

\subsection{Data propagation}

We next describe how the data are disseminated through a network. We have two implementation strategies for data propagation. One is gradient-based and the other is broadcast-based.

\subsubsection{Gradient-based}

In the interest broadcast phase, when a node receives an interest from the neighboring nodes with a stronger magnetic charge, the node will establish a gradient toward the interest-sending node. This gradient will lead the data through the network from the source to the sink.

When a node senses data, it checks if it has an entry matching the data type. If it does, the node sends the data to the nodes pointed by the existing gradients. The receiving nodes check for the matching entry and then send the a

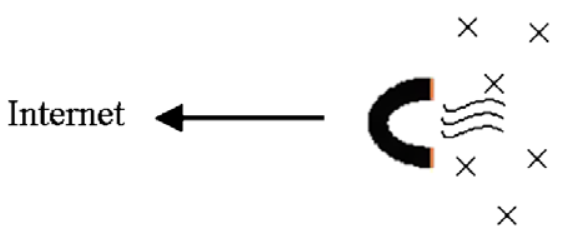

b

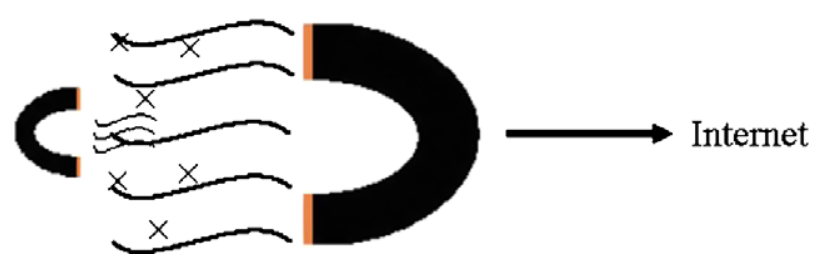

Fig. 4. Resource awareness: larger magnet out-attracting the small magnet. (a) Small magnet (resource-limited sink, e.g., PDA) attracts data and transmits via resource-limited network (e.g., GPRS) to Internet. (b) Large magnet (more resourceful sink, e.g., PC) which overwhelms the small magnet, attracts data, and transmits via wider bandwidth network (e.g., ADSL) to Internet. 

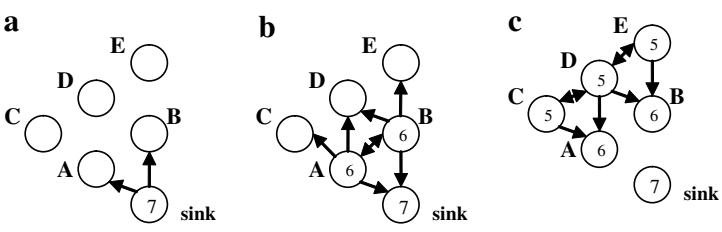

Fig. 5. An example of interest propagation.

data according to the gradients as well. The forwarding process goes on and the data will reach the sink eventually.

Fig. 6(a) shows the gradients established by the interest broadcast in Fig. 5. In Fig. 6(b), the source sends data to the two neighbor nodes pointed by the gradients from the source. In Fig. 6(c), the receiving nodes continue to send data to their neighbor nodes based on their gradients. The sink finally receives the data.

\subsubsection{Broadcast-based}

In the broadcast-based mode, the nodes do not establish gradients in the interest broadcast phase. Instead, the magnetic charge is included in the data being disseminated. The receiving node can tell from the charge carried in the data where the data are coming from and whether to forward the data further down the stream.

More specifically, when a node receives the data, the node checks if it has any matching entry. If so, it compares the magnetic charge in the entry with the magnetic charge of the data. If the magnetic charge in the entry is greater than that of the data, it sets the magnetic charge of the data to the charge in the entry and then broadcasts the data. This means the data are sent from the node whose magnetic charge is lower than the intermediate node and the intermediate node should continue to broadcast them toward the sink. If a node receives a duplicate data or the data whose magnetic charge is greater than that in the entry, the data will be discarded.

This dissemination mode is easy to implement and we do not need to maintain the gradients. The node simply compares the magnetic charges to decide whether to broadcast the data. The drawback is that every data message will have to carry the magnetic charge of the sending node.

In Fig. 7(a), the magnetic charge of every node is established with the sink of change strength 7. In Fig. 7(b), when the source wants to send data to the sink, it will broadcast the data to its neighbor nodes. In Fig. 7(c), the nodes with charge strength 6 broadcast the data because the magnetic
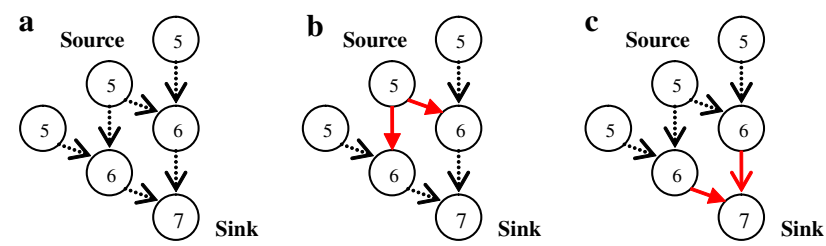

Fig. 6. An example of data propagation with gradient-based mechanism.
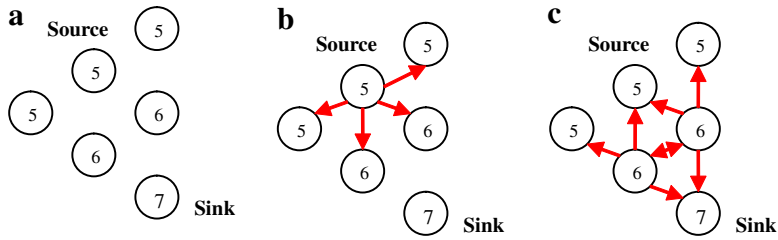

Fig. 7. An example of data propagation with broadcast-based mechanism.

charge of the nodes is greater than that of the data. Thus, the sink receives the data.

\subsection{Random wait}

When the interests or data are being broadcast hop by hop through the network, the messages might collide to each other. To avoid collisions, the node waits a random period of time before sending a message. This technique might decrease the probability of collision and, in the meantime, increase the transmission delay.

\section{Evaluation}

We have conducted an extensive set of simulations that examines the scalability of MD to the size of the network, the change of the data rate, and the level of the network dynamics. In Section 4.1, we describe the simulation setup and the metrics examined for the performance evaluation. In Section 4.2, we provide the comparisons of two different mechanisms of MD. Section 4.3 examines the scalability of MD. The impact of random wait to the reliability of MD is discussed throughout Section 4.4. In Section 4.5, we focus on the quality of data delivery and the message overhead of MD in the presence of network dynamics. In Section 4.6, a set of selection guidelines for data dissemination mechanisms is provided.

\subsection{Simulation setup and metrics}

In this section, we describe the simulation setup and the metrics examined for the performance evaluation. We implemented MD in the ns-2 simulator [30]. In order to get the average behavior, there are 10 distinct runs for each setup. In each run, there are 50 nodes randomly placed in a $160 \mathrm{~m}$ by $160 \mathrm{~m}$ square. Each node has a radio range of $40 \mathrm{~m}$. We use one source and one sink randomly selected from 50 nodes in our simulation. The source sends data every $5 \mathrm{~s}$, and the interests are periodically sent every $30 \mathrm{~s}$. The simulation time is $600 \mathrm{~s}$, but the source will stop sending data after $300 \mathrm{~s}$. Table 1 summarizes the parameters of our choice.

The above scenario serves as the base setup. For the comparisons of gradient-based and broadcast-based MD, we use the exact base setup. To examine the scalability of the mechanisms, we increase the number of nodes from 10 to 200 . The size of the simulated area is increased to 
Table 1

Simulation settings

\begin{tabular}{ll}
\hline Nodes & 50 \\
Size & $160 \times 160$ \\
Radio range & $40 \mathrm{~m}$ \\
Data rate & $5 \mathrm{~s}$ \\
Periodic interest & $30 \mathrm{~s}$ \\
MAC & 802.11 \\
Antenna & OmniAntenna \\
Simulation time & $600 \mathrm{~s}$ \\
\hline
\end{tabular}

keep the node density constant. To study the impact of random wait, we keep all parameters the same as in the base setup except for the range of the random wait intervals. To simulate network dynamics, the nodes are scheduled to move around in the simulated area and the nodes may go on and off in random patterns.

We choose three metrics to evaluate the performance of MD and to compare it to other schemes: Overhead measures the amount of interest and data packets transmitted. The metric is closely related to the energy consumption of the system in wireless sensor networks when an energy-efficient MAC protocol is applied. Reachability measures the probability the sink receives the data packet successfully. This metric is important for medical applications in that the sensor data are mission critical and data losses can be life-or-death matters. Latency measures the data transmission time from the source to the sink. For medical applications, the metric represents the timeliness and temporal reliability of the data.

\subsection{Gradient vs. broadcast}

To compare the performance of two forwarding strategies, we simulate both the MD gradient (MDG) and broadcast (MDB) modes using the basic setup. Simulation results are shown in Table 2 and Fig. 8. Table 2 shows the overhead in terms of the data, interest, total packet counts, and the average reachability. Fig. 8 depicts the cumulative distribution of the data delivery latency. The data latency of MDB is generally better than that of MDG. As the results suggest, there are several advantages of the MDB mode over the MDG mode. MDB is more energy efficient. The interest packet overhead of the two modes is the same because interests are transmitted in an identical way in both modes. The difference lies in the data packet overhead. In MDG, if a node has five gradients to its neighbors,

Table 2

Comparisons of overhead and reachability between MDG and MDB

\begin{tabular}{lcl}
\hline & Gradient & Broadcast \\
\hline Interest packets & 4,970 & 4970 \\
Data packets & 5,162 & 3490 \\
Total packets & 10,132 & 8460 \\
Reachability & $100 \%$ & $98.6441 \%$ \\
\hline
\end{tabular}

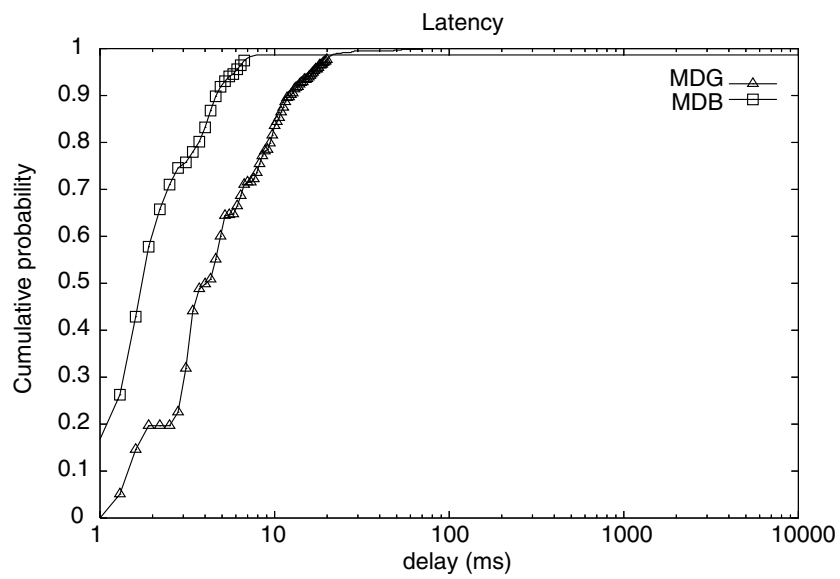

Fig. 8. Cumulative distribution of delay time for MDG and MDB.

there are five packets sent, one for each gradient. But in MDB, it broadcasts only one data packet. Therefore, the overall overhead of MDB is much smaller than MDG. Additionally, the data latency is small in MDB. It does not need to send the handshake packets, e.g., the RTS/ CTS in IEEE 802.11, before sending a data packet, but MDG does. As a result, MDB behaves better in latency. However, without the handshaking, there are more collisions observed in MDB simulations, and this results in a slight lower reachability in some cases. MDB also performs better in the presence of network dynamics. Given the various advantages of the MDB over MDG, we use MDB for the rest of the comparison.

\subsection{Scalability}

To compare the scalability of DD, MD, and flooding, we change the number of nodes in the simulated network from $10,50,100,150$, to 200 . We change the size of the simulated area accordingly to keep a fixed node density.

\subsubsection{Overhead}

Fig. 9 shows the overhead for different number of nodes. In Fig. 9(a), the interest packet overhead of TPP is slightly higher than that of OPP and MD in all cases. This is because TPP has to disseminate additional control packets such as the positive and negative reinforcement messages, whereas MD and OPP do not.

Note that we set the interval of periodic interest to $30 \mathrm{~s}$ in all mechanisms. That is the reason that the interest packet overhead of MD and OPP is almost identical.

Fig. 9(b) shows that MD has a lower data packet overhead than that of the TPP and a higher data overhead over that of the OPP in all network sizes except the 10-node case. OPP selects only one way to disseminate data, and thus has the least amount of data packet overhead. This result suggests that, independent of the network sizes, the resulting data packet overhead of TPP's exploratory data is higher than that of MD's multi-path delivery, and that MD is more energy efficient than TPP. In the 10-node case, 
a

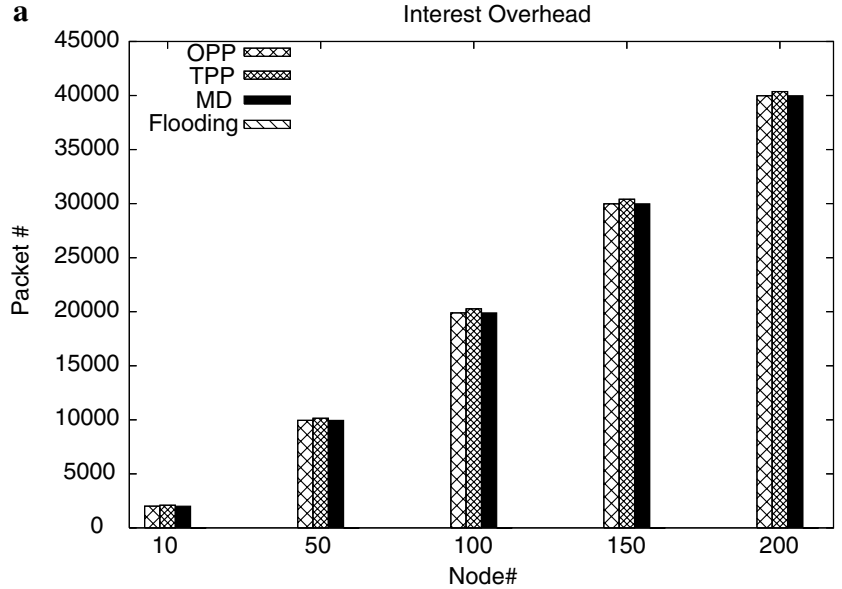

b

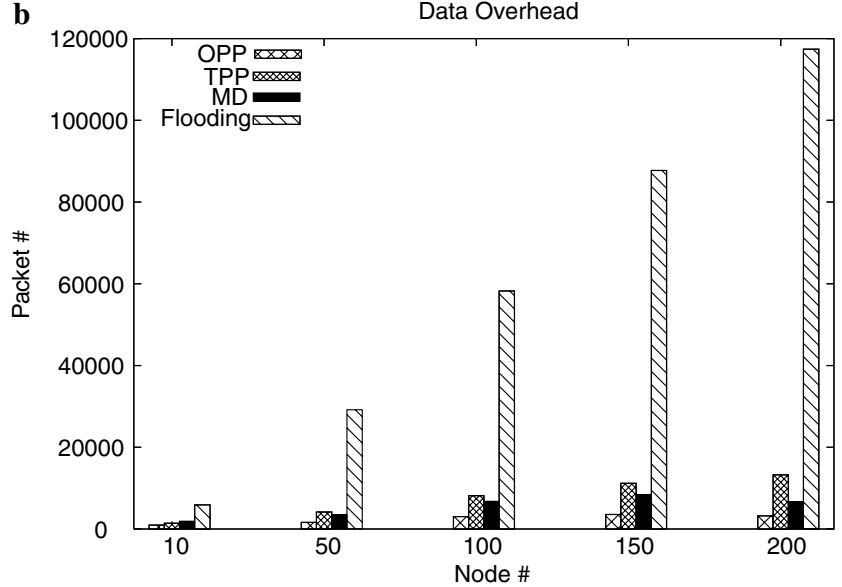

c

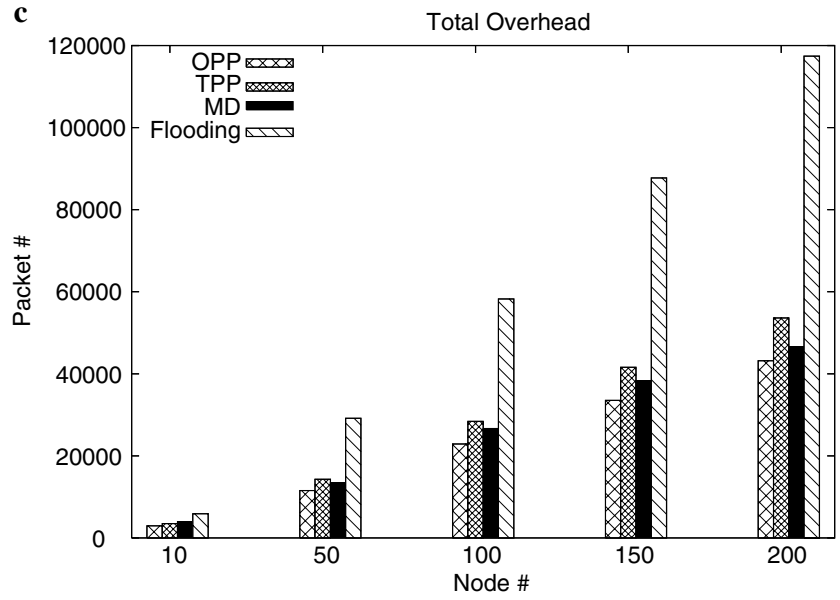

Fig. 9. Overhead with increasing network sizes. (a) Interest packet overhead. (b) Data packet overhead. (c) Total (interest + data) packet overhead.

MD's multiple paths might cover the whole network and thus the data packet overhead of MD and TPP is too close to set the results apart. It seems that MD is worse than OPP in terms of data packet overhead. However, the slightly higher data overhead, in return, provides better data reachability and latency. The reachability and latency of the mechanisms will be discussed next.
Fig. 9(c) shows that flooding has the largest total packet overhead even though the mechanism does not require any control packets. More importantly, we observe that (1) flooding scales the worst with the increasing network size, and (2) the multi-path MD scales as well as the single-path OPP.

\subsubsection{Reachability}

Fig. 10 shows the reachability of the mechanism in different network sizes. OPP and TPP experience an $100 \%$ reachability, MD and flooding's reachability is at about $95 \%$. The reason of the difference is at the way the data are propagated. OPP and TPP are gradientbased, whereas MD and flooding are broadcast-based. If data packets collide, the gradient-based mechanisms will retransmit assuming IEEE 802.11 as the underlying MAC protocol. The broadcast-based mechanisms, however, do not detect, nor recover for errors, and thus the slightly lower reachability observed in MD and flooding. Reachability of the mechanisms seems to be independent of the network size.

\subsubsection{Latency}

The latency results are similar with varying network sizes. We compare the mechanisms first using the 50-node result and then discuss their scalability with increasing network sizes. In Fig. 11, MD has the least latency because it broadcasts data and bypasses the RTS/CST handshake in IEEE 802.11. This is also why OPP performs much worse than MD, even if it always finds the fastest path. Flooding is better than OPP and TPP only in the preceding $60 \%$. We observe an unusual amount of traffic and collisions in the flooding set of simulations. As a result, some data packets get retransmitted repeatedly and routed for a long way to finally reach the sink. This explains why flooding has a lot of data packets with long latency. TPP is very close to OPP in the preceding $90 \%$, but there is a long tail caused by the random wait mechanism in disseminating exploratory data in the later $10 \%$. This plot shows that the broad-

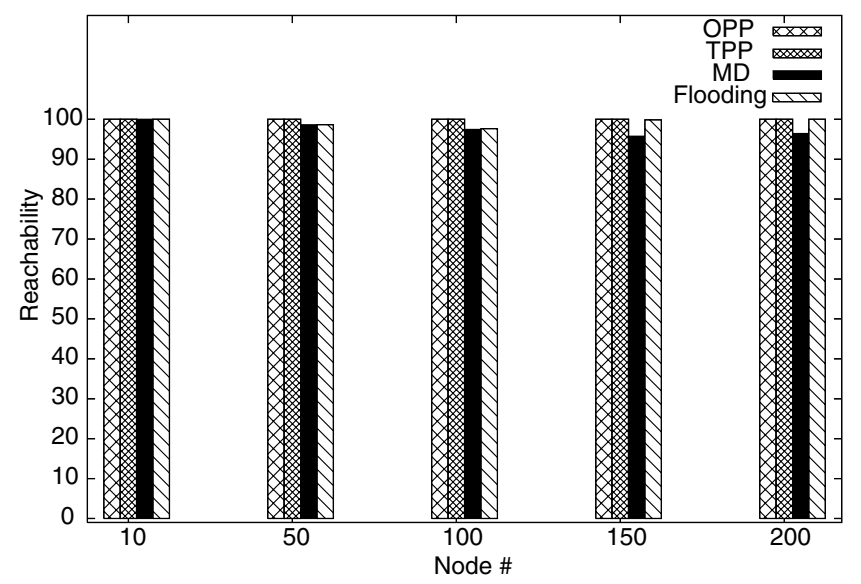

Fig. 10. Reachability for 10-, 50-, 100-, 150-, and 200-node networks. 


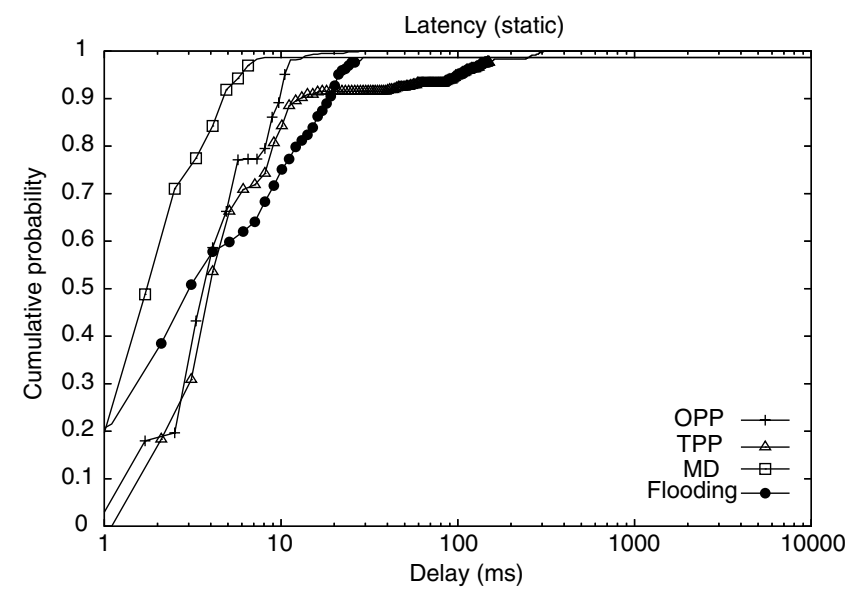

Fig. 11. Cumulative distribution of delay time for DD, MD, and flooding.

cast-based mechanisms work better for applications with latency requirements.

For other network sizes, we find all curves shift to the right. The reason is that the transmission paths are generally longer when the networks are larger. The advantage of flooding over OPP and TPP diminishes with the increasing number of nodes. More nodes generate more traffic. This results in more collisions consequently. Thus, there are gradually more packets experiencing long delays in the flooding case. MD scales better than flooding by propagating data over a selective set of paths.

\subsection{Impact of random wait}

The slight lower reachability in MD and flooding is due to collisions. As opposed to the gradient-based DD where point-to-point packet losses are detected and recovered by the underlying MAC protocol, broadcastbased MD and flooding will have to avoid collisions to reduce the amount of packet losses. In view of improving the reachability, we implement a random wait mechanism. Every node waits for a random amount of time, uniformly selected within a pre-defined range, before sending any broadcast-based packets. This mechanism avoids the sending of packets simultaneously and thus will be able to reduce the amount of collisions experienced by MD and flooding.

\subsubsection{Collision}

Fig. 12 shows the number of collisions for different random wait ranges. The number of collisions drops drastically as the range increases, but the drop slows down when the range goes beyond 4-6 ms. A larger range implies a larger average waiting time. Having the packets waiting longer in the network will result in a higher degree of end-to-end latency. That is, a smaller range is preferred for a lower overall packet delay. This concave curve suggests that most of the collisions can be avoided with a small range, and there is little marginal benefit to use a large range.

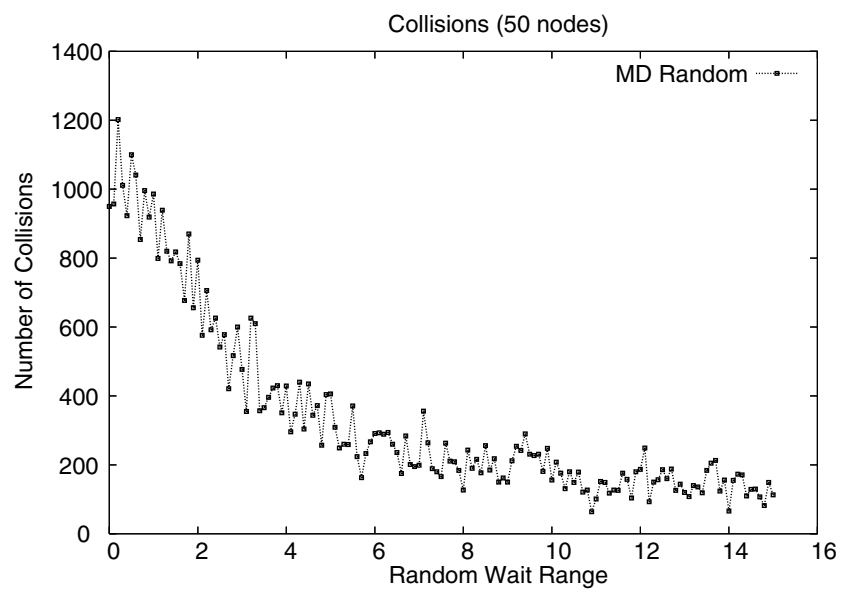

Fig. 12. Number of collisions with increasing random wait range.

\subsubsection{Reachability}

Fig. 13 shows the reachability for applying different ranges to the random wait mechanism. The reachability rises rapidly within $4 \mathrm{~ms}$, and then remains steady in between $99.8 \%$ and $100 \%$. The result echoes the discussion about collisions. We see the number of collisions drops significantly for the first $4 \mathrm{~ms}$, and this is exactly where the reachability rises most evidently. This convex curve suggests that the reachability can be improved to pseudo $100 \%$ with a small random wait range, and there is no need of using a larger range. Four milliseconds is empirically the optimal range to use for this set of simulated networks. Given the rate of collision depends on the number of nodes sharing the wireless medium, we think the optimal range value depends on the network density.

\subsubsection{Data with acceptable delay}

Fig. 14 shows the percentage of data packets with lower than $50-\mathrm{ms}$ end-to-end delay. The reason that we choose $50 \mathrm{~ms}$ to be the threshold is that 50 -ms delay is too short to be distinguishable by human. In other words, data arriving with an extra 50-ms delay are acceptable. The 50-ms

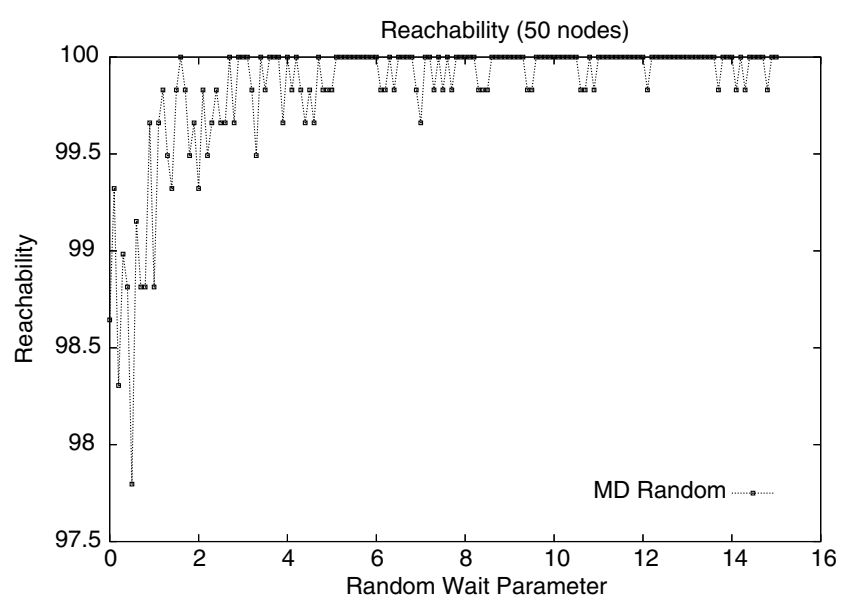

Fig. 13. Reachability with increasing random wait range. 


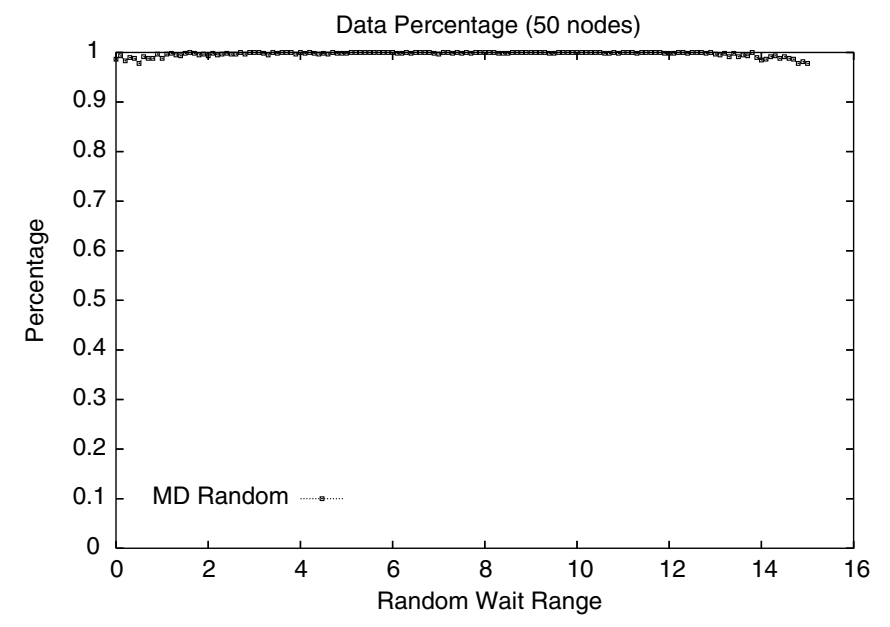

Fig. 14. Percentage of data packets arriving within $50 \mathrm{~ms}$ with increasing random wait range.

data arrival rates are all very close to $100 \%$ using the random wait mechanism. This suggests that the random wait mechanism is effective in improving the reachability of MD and the extra delay is too small to be perceptible.

\subsection{Impact of dynamics}

To be realistic, we simulated two kinds of dynamics in our experiments, mobile and node on-off. For the mobile simulations, each node moves at $120 \mathrm{~s}$. After a node moves to a new random position, it waits another $120 \mathrm{~s}$ to move again. The amount of time that a node takes to move to its new position varies from node to node because of the random new position selection. As a result, the movements of the nodes are not simultaneous.

In the node on-off simulations, each node is turned on and off according to the following probabilistic distribution. At the beginning, all nodes are in the on state for a random period between 5 and $65 \mathrm{~s}$. Then, each node goes into the off state for $25-35 \mathrm{~s}$, and wakes up again for 55 $65 \mathrm{~s}$. All the on or off time durations are uniform randomly selected and this process will continue until the end of the simulations.

These sets of simulations are necessary because the two types of dynamics are common in reality. The mobile type emulates people maneuvering in office buildings where we move, stop to mind some business, and then move again. The on-off type represents sensor nodes operating continuously for some time, being stopped for some time to change for new batteries, and then resuming the operation. The choices of the parameters represent the extreme cases to highlight the distinct properties in MD to the other two mechanisms, DD and flooding.

\subsubsection{Overhead}

Fig. 15 shows the amount of overhead for the static, mobile, and on-off cases. Note that in the static case, all nodes remain static and on for the entire duration of the
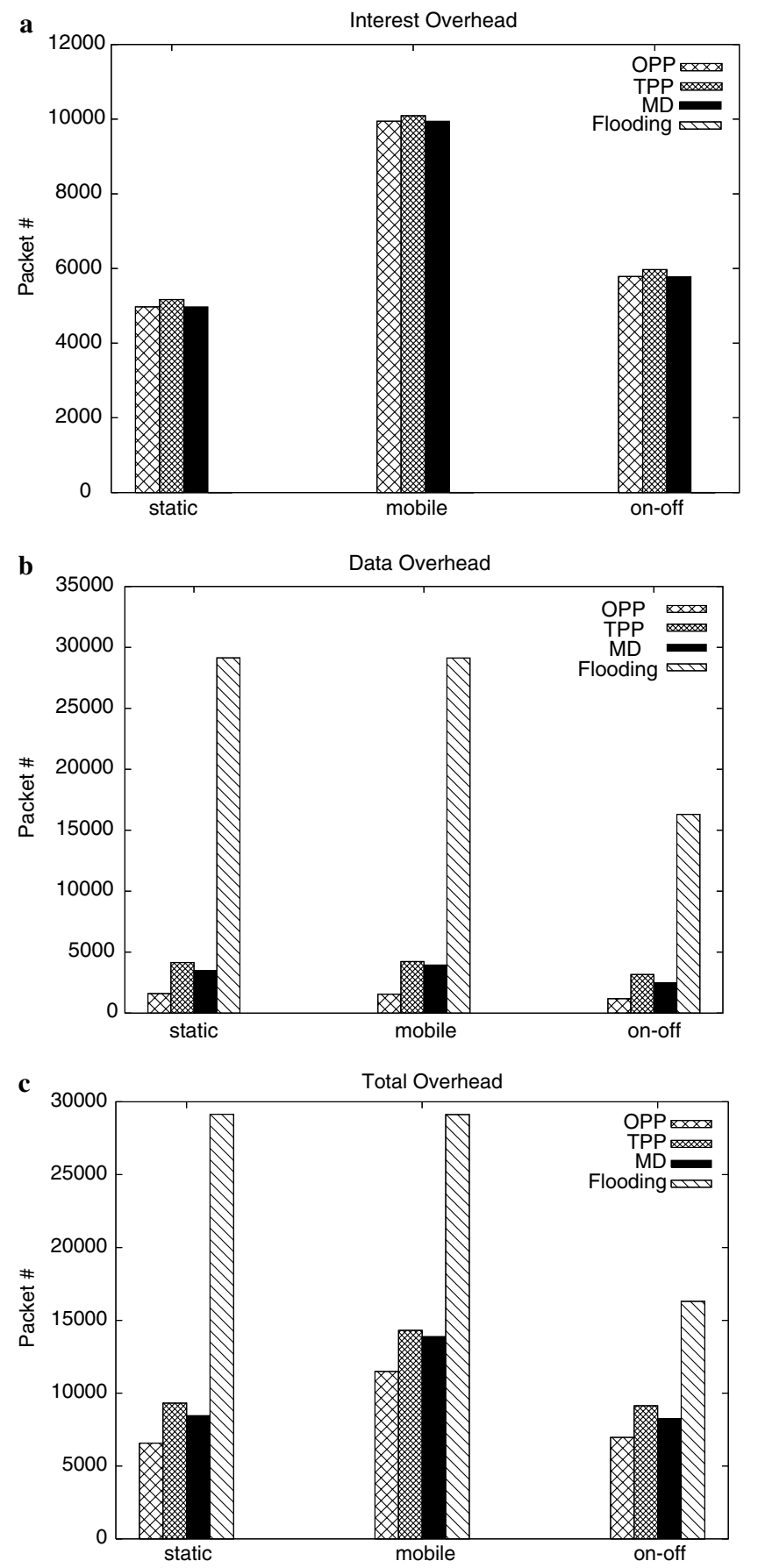

Fig. 15. Overhead for static, mobile, and on-off cases. (a) Interest packet overhead. (b) Data packet overhead. (c) Total (interest + data) packet overhead.

simulations. Figs. 15(a)-(c) show the amount of interest, data, and total packets, respectively. From Fig. 15(c), we observe the general trend of flooding experiencing a higher amount of packet overhead in all three cases. The total packet overhead of DD and MD is higher in the mobile case due to the increase in the interest packet overhead (Fig. 15(a)). The DD- or MD-related states in the mobile nodes may be changed as the nodes move. These changes will be propagated by means of the interest or other control 
packets which results in a higher amount of interest overhead. Fig. 15(b) shows that the data packet overhead of the on-off case is lower. This is because certain critical nodes in the network might be turned off and a significant amount of data fails to traverse through the network.

\subsubsection{Reachability}

Fig. 16 shows the reachability results of the static, mobile, and on-off cases. In the mobile case, the reachabilities of OPP and TPP drop significantly. MD's reachability is reduced to $85 \%$, which is about $20 \%$ higher than TPP (65\%) and 10\% higher than OPP (75\%). This is because MD sends data in multiple paths. When the nodes are mobile, the probability is higher to get the data through the network to the sink with multiple paths.

Note that TPP performs worse than OPP in the mobile case. The difference lies in the path update frequency. OPP reconfigures data path at the time of periodic interest, but TPP does so at the time of periodic exploratory data. The interval of periodic exploratory data is twice as much as the periodic interest. Therefore, TPP results in a lowest reachability in this case. If we shortened the time of periodic exploratory data, there will be a higher reachability, but the data packet overhead will also increase. From this set of results, we can see the advantages of MD over OPP and TPP in terms of reachability in mobile sensor networks. Note that the reachability of flooding is close to $100 \%$. The result suggests that if the data are absolutely critical and energy is not a constraint, flooding is the best choice for reliable data dissemination. Otherwise, MD provides as a reliable solution for environment that energy resource is limited.

In the on-off case, we observe similar results. But the performance is unsatisfactory. Even the flooding mechanism manages only $85 \%$ reachability. This is because of the potential of broken paths, or even a disconnected network, when certain bottleneck nodes are turned off. Such conditions result in a lower overall reachability.

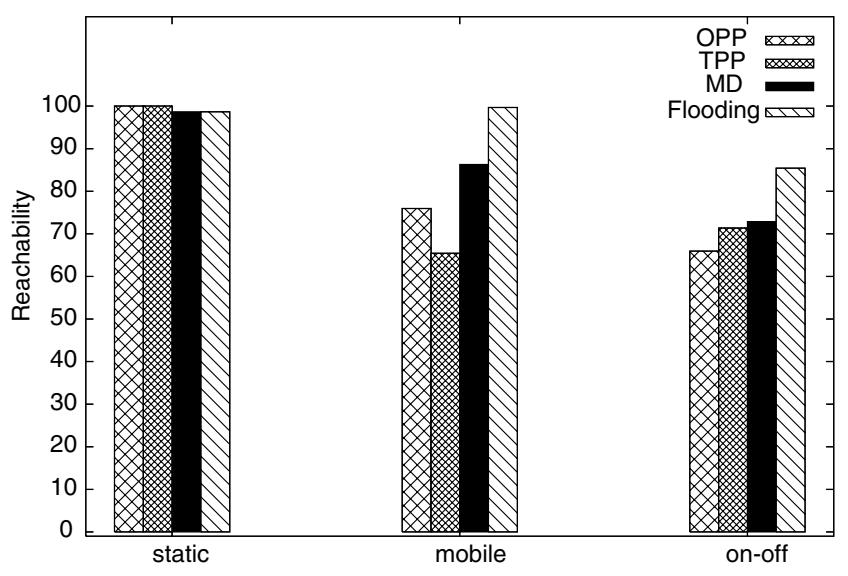

Fig. 16. Reachability for static, mobile, and on-off case.
However, we think that a good deployment strategy may compensate for such situations. We find that OPP performs the worst in the on-off case. In some sense, the on-off type dynamic presents greater challenge to reliable delivery of data and OPP, or single-path mechanisms, is less suitable for networks with extreme dynamics.

\subsubsection{Latency}

Fig. 17 shows the cumulative probability of data delivery latency for the static, mobile, and on-off cases. The static case result, Fig. 17(a), is the same as Fig. 11 shown in Section 4.3. The figure is presented again here only for the reference. We observe, in Fig. 17(b), that MD has the least degree of latency yet. Note that flooding performs better than OPP completely. This is because OPP has to retransmit a packet many times as a result of high frequency of packet loss and collision in mobile networks. The same reason in OPP and lost data packets result in the poor performance of TPP. Fig. 17(c) shows similar result as well. These results indicate that $\mathrm{MD}$ is a better solution for applications with requirements of restricted latency in dynamic network.

\subsection{Summary and selection guidelines}

Tables 3 and 4 summarize our comparison of MD, DD, and flooding in the static and dynamic cases. Note that the term DD here refers to the best of OPP and TPP in each case. We find that the choice of data dissemination mechanism is application requirement and deployment environment dependent. Based on the experimental results, we are able to derive the following selection guidelines:

- For static and stable wireless sensor networks, DD is the best choice.

- For dynamic wireless sensor networks with energy constraint, MD is a more plausible solution with a balanced performance in energy efficiency, reliability, and data latency.

- For networks that require absolute reliability, flooding with continuous power supply is a better option.

\section{Conclusion}

Inspired by the physics of magnets and nails, we propose magnetic diffusion, a simple and yet efficient dissemination mechanism for mission-critical data. MD is able to identify all the shortest paths available from the data source to sink. By transmitting data over the multiple shortest paths, MD performs well in the timeliness and reliability of data delivery while the overhead and energy consumption of $\mathrm{MD}$ is kept low. These properties are confirmed by the simulations. Therefore, we conclude that MD is particularly suitable for sensor network applications 

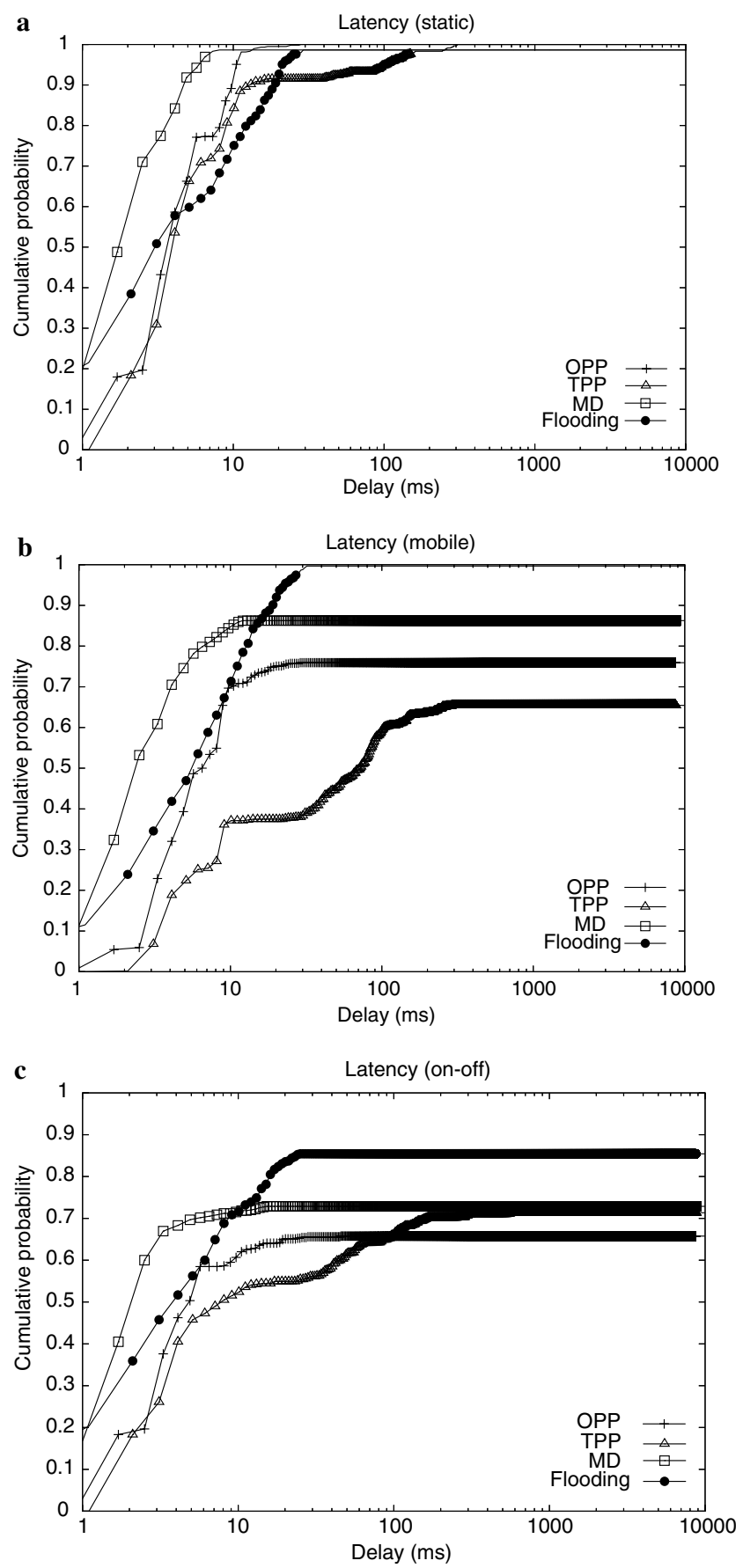

Fig. 17. Cumulative distribution of delay time for (a) static, (b) mobile, and (c) on-off cases.

Table 3

Comparison of MD, DD, and flooding in static case

\begin{tabular}{llll}
\hline & Best & Fair & Worst \\
\hline Overhead & DD & MD & Flooding \\
Reliability & DD & Flooding & MD \\
Latency & MD & DD & Flooding \\
\hline
\end{tabular}

in healthcare and workplace safety. For these applications, the timeliness and reliability of data, and the energy efficiency of the system are all required properties.
Table 4

Comparison of $\mathrm{MD}, \mathrm{DD}$, and flooding in dynamic case

\begin{tabular}{llll}
\hline & Best & Fair & Worst \\
\hline Overhead & DD & MD & Flooding \\
Reliability & Flooding & MD & DD \\
Latency & MD & Flooding & DD \\
\hline
\end{tabular}

\section{Acknowledgments}

This work is sponsored by National Science Council of Taiwan under Contract NSC-93-2218-E-002-147, NSC-932218-E-002-144, and NSC-93-2213-E-002-121. The authors would also like to acknowledge the support from the Intel Digital Health Center grant.

\section{References}

[1] Chalermek Intanagonwiwat, Ramesh Govindan, Deborah Estrin, Directed diffusion: a scalable and robust communication paradigm for sensor network, in: Proceedings of the Sixth Annual International Conference on Mobile Computing and Networking (MobiCOM '00), August 2000, Boston, MA.

[2] John Heidemann, Fabio Silva, Deborah Estrin, Matching data dissemination algorithms to application requirements, in: Proceedings of the ACM SenSys Conference, Los Angeles, California, USA, ACM, New York, 2003, pp. 218-229.

[4] Wendi Rabiner Heinzelman, Anantha Chandrakasan, Hari Balakrishnan, Energy-efficient communication protocol for wireless microsensor networks, in: Proceedings of the 33rd Hawaii International Conference on System Sciences, vol. 8.

[5] Seema Bandyopadhyay, Edward Coyle, An energy-efficient hierarchical clustering algorithm for wireless ad hoc networks, in: Proceedings of the 22nd Annual Joint Conference of the IEEE Computer and Communications Societies (INFOCOM), 2003.

[6] Ossama Younis, Sonia Fahmy, Distributed clustering in ad-hoc sensor networks: a hybrid, energy-efficient approach, in: Proceedings of the 23nd Annual Joint Conference of the IEEE Computer and Communications Societies (INFOCOM), 2004.

[7] S.D. Servetto, G. Barrenechea, Constrained random walks on random graphs: routing algorithms for large scale wireless sensor networks, in: Proceedings of the 1st AC International Workshop on Wireless Sensor Networks and Applications (WSNA), Atlanta, GA, September 2002.

[8] David Braginsky, Deborah Estrin, Rumor routing algorithm for sensor networks, in: Proceedings of the 1st AC International Workshop on Wireless Sensor Networks and Applications (WSNA), Atlanta, GA, September 2002.

[9] Yan Yu, Ramesh Govindan, Deborah Estrin, Geographical and Energy Aware Routing: a recursive data dissemination protocol for wireless sensor networks, Technical Report UCLA/CSD-TR-01-0023, UCLA Computer Science Department, May 2001.

[10] Ananth Rao, Christos Papadimitriou, Scott Shenker, Ion Stoica, Geographic routing without location information, in: Proceedings of the 9th Annual International Conference on Mobile Computing and Networking, San Diego, CA, USA, September 14-19, 2003.

[11] Hyung Seok Kim, Tarek F. Abdelzaher, Wook Hyun Kwon, Minimum-energy asynchronous dissemination to mobile sinks in wireless sensor networks, in: Proceedings of the 1st International Conference on Embedded Networked Sensor Systems, Los Angeles, California, USA, November 05-07, 2003.

[12] CFan Ye, Haiyun Luo, Jerry Cheng, Songwu Lu, Lixia Zhang, A two-tier data dissemination model for large-scale wireless sensor networks, in: Proceedings of the 8th Annual International Conference on Mobile Computing and Networking, Atlanta, Georgia, USA, September 23-28, 2002. 
[13] Chieh-Yih Wan, Andrew T. Campbell, Lakshman Krishnamurthy, PSFQ: a reliable transport protocol for wireless sensor networks, in: Proceedings of the 1st ACM International Workshop on Wireless Sensor Networks and Applications, WSNA 2002, September 2002.

[14] Y. Sankarasubramaniam, O.B. Akan, I.F. Akyildiz, ESRT: Event-tosink reliable transport in wireless sensor networks, in: Proceedings of the ACM MobiHoc'03, Annapolis, Maryland, USA, June 2003.

[15] Omprakash Gnawali, Mark Yarvis, John Heidemann, Ramesh Govindan, Interaction of retransmission, blacklisting, and routing metrics for reliability in sensor network routing, in: Proceedings of the First IEEE International Conference on Sensor and Ad hoc Communications and Networks, SECON, 2004.

[16] Sukun Kim, R. Fonseca, D. Culler, Reliable transfer on wireless sensor networks, in: Proceedings of the First IEEE International Conference on Sensor and Ad hoc Communications and Networks, SECON, 2004.

[17] Bret Hull, Kyle Jamieson, Hari Balakrishnan, Techniques for mitigating congestion in sensor networks, in: Proceedings of the ACM SenSys 2004, Baltimore, MD, November 2004.

[18] Chieh-Yi Wan, Shane B. Eisenman, Andrew T. Campbell, CODA: COngestion detection and avoidance in sensor networks, in: Proceedings of First ACM Conference on Embedded Networked Sensor Systems, SenSys 2003, Los Angeles, November 5-7, 2003, pp. 266-279.

[19] Yaling Yang, R. Kravets, Distributed QoS guarantees for realtime traffic in ad hoc networks, in: Proceedings of the First IEEE International Conference on Sensor and Ad hoc Communications and Networks, SECON, 2004

[20] Douglas De Couto, Daniel Aguayo, John Bicket, Robert Morris, A high-throughput path metric for multi-hop wireless routing, in: Proceedings of the 9th Annual International Conference on Mobile Computing and Networking, MobiCom 2003, September 2003, pp. 134-146.

[21] Baruch Awerbuch, David Holmer, Herbert Rubens, High throughput route selection in multi-rate ad hoc wireless networks, in: Proceedings of the Wireless On-Demand Network Systems, First IFIP TC6 Working Conference, WONS 2004, January 21-23, 2004.

[22] S.R. Medidi, K. Vik, QoS aware source-initiated ad-hoc routing, in: Proceedings of the First IEEE International Conference on Sensor and Ad hoc Communications and Networks, Santa Clara, CA, USA, October 2004.

[23] Budhaditya Deb, Sudeept Bhatnagar, Badri Nath, ReInForM: Reliable information forwarding using multiple paths in sensor networks, in: 28th Annual Conference on Local Computer Networks, LCN, 2003.

[28] J.M. Kahn, R.H. Katz, K.S.J. Pister, Mobile networking for smart dust, in: Proceedings of the ACM/IEEE International Conference on Mobile Computing and Networking (MobiCom99), Seattle, WA, August 17-19, 1999.

[29] J. Hill, R. Szewczyk, A. Woo, S. Hollar, D. Culler, K. Pister, System architecture directions for networked sensors, in: ACM SIGOPS Operating Systems Review, vol. 34, No. 5, 2000, pp. 93-104.

[30] L. Breslau, D. Estrin, K. Fall, S. Floyd, J. Heidemann, A. Helmy, P. Huang, S. McCanne, K. Varadhan, Y. Xu, H. Yu, The VINT Project, Advances in network simulation, IEEE Computer 33 (5) 2000 59-67.

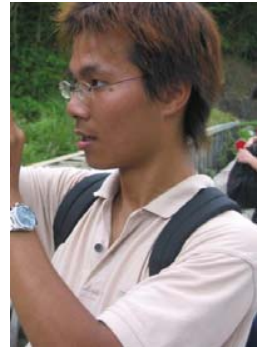

Hsing-jung Huang received the B.S. degree in Computer Science and Information Engineering from National Taiwan University, Taiwan, in 2004. He is currently a graduate student of Department of Electrical Engineering of National Taiwan University. His research activity mainly focuses on routing protocols in wireless sensor networks.

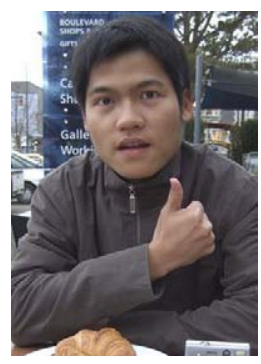

Ting-Hao Chang received the B.S. degree in Computer Science and Information Engineering from National Taiwan University, Taiwan, in 2004. He is currently a graduate student of Department of Electrical Engineering of National Taiwan University. His research activity mainly focuses on reliable transfer over wireless sensor networks.

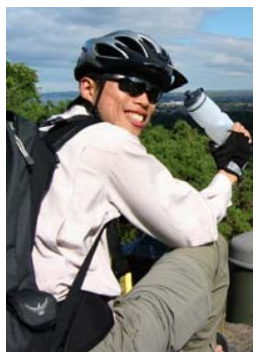

Shu-Yu Hu received the B.S. degree in Computer Science and Information Engineering from National Taiwan University (NTU), Taiwan, in 2004. He is currently a graduate student of Department of Electrical Engineering of the National Taiwan University. His research activity mainly focuses on sensor network deployment issue.

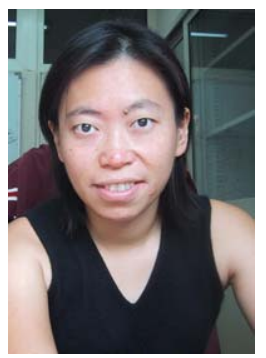

Polly Huang received her Ph.D. (1999) and M.S. (1997) in Computer Science from University of Southern California, and her B.S. (1993) in Mathematics from National Taiwan University. In February 2003, she joined the Department of Electrical Engineering of the National Taiwan University at which she currently holds an assistant professor position. Prior to joining NTU, she worked as a post-doctoral research scientist at the Computer Engineering and Networks Laboratory (TIK) of the Swiss Federal Institute of Technology (ETH) Zurich. Dr. Huang is on the editorial board of Journal of Communications and Networks. She is a member of the ACM and IEEE. 